\title{
Computational approaches to the art music traditions of India and Turkey
}

\author{
Xavier Serra \\ Music Technology Group, Universitat Pompeu Fabra, Barcelona
}

[This is an Author's Original Manuscript of an Article whose final and definitive form, the Version of Record, has been published in the Journal of New Music Research, Volume 43, Issue 1, 1-2, 31 Mar 2014, available online at: http://dx.doi.org/10.1080/09298215.2014.894083]

This special issue has its origins in the $2^{\text {nd }}$ CompMusic workshop that took place in Istanbul (Turkey) on July $12^{\text {th }}$ and $13^{\text {th }} 2012$. The workshop included 28 talks, most of them on research carried out within CompMusic but it also had presentations by other researchers working on topics of relevance to the project. After the workshop, the scientific committee made a selection of the best presentations and the authors were asked to write and submit extended articles to this special issue. After another review and selection process we have been able to put together the eight articles published here.

CompMusic is a research project funded by the European Research Council that aims to advance in the field of music information research by emphasizing cultural specificity. It carries research on the automatic description of music by focusing on five music traditions: Hindustani (North India), Carnatic (South India), Turkish-makam (Turkey), Arab-Andalusian (Maghreb), and Beijing Opera (China). During the first part of the project the musics of India and Turkey were the most studied ones and both the workshop in Istanbul and this special issue reflect that focus.

Despite recent efforts by the music information research (MIR) community to open up towards non-western music, most current research is centered on a few aspects of the western commercial music of the past few decades. This music repertoire conditions the problems being worked on and thus the solutions obtained. If we open up to other types of music we should discover new interesting problems to be solved that require new methodologies and new solutions.

For the development of cultural specific music information processing methods that can challenge the dominant western-centered information paradigms, it is of great advantage to work with classical musical cultures for which there is a relevant body of musicological and cultural studies available. If we are able to take advantage of existing formalizations of a music repertoire we can more easily develop musically meaningful computational and information models. This has been the basic hypothesis of CompMusic.

The Turkish-makam and the Indian art music traditions, Hindustani and Carnatic, are very suitable musical repertoires with which to evaluate the limitations of current computational music methodologies and with which to explore new research directions. The articles presented in this issue are excellent contributions in this direction. This compilation of articles has aimed to overview relevant musical formalisms of these music traditions, to review the state of the art in music information research that has focused on these music repertoires, to present new research results in the analysis of the 
characteristic melodic and rhythmic aspects of these musics, and finally to identify research directions of relevance to the music information research field.

With respect to Turkish makam music, the article by B. Bozkurt, R. Ayangil, A. Holzapfel reviews the musical concepts of relevance to computational work and summarizes the state of the art and current challenges related to that. The article by E. Unal, B. Bozkurt and M. K. Karaosmanoğlu is a contribution to the melodic analysis of makam music by using a large and representative collection of machine-readable musical scores and proposing a method for the classification of makams. The article by S. Şentürk, A. Holzapfel and X. Serra focuses on the linking musically relevant sections of scores and audio recordings, two needed complementary sources of information of relevance to study melodic and rhythmic aspects of this music. Finally the article by A. Srinivasamurthy, A. Holzapfel and X. Serra presents their research on the analysis of rhythm from audio recordings for both makam and Indian music. It evaluates current methodologies used in MIR for different rhythm description tasks, identifying their shortcoming and justifying the need for developing specific methods to analyze these musics.

With respect to Indian music, the article by S. Rao and P. Rao offers an excellent musicological and computational perspective to Hindustani music, reviewing the musical concepts mainly related to the melodic aspects of this music tradition and identifying relevant research problems to be addressed computationally. The article by S. Gulati, A. Bellur, J. Salamon, Ranjani H. G., V. Ishwar and X. Serra is a comprehensive overview of all the research done on tonic identification specific for indian music, doing a comparative evaluation of all the existing approaches. The article by G. K. Koduri, J. Serrà and $\mathrm{X}$. Serra studies the issue of intonation specific for Carnatic music, developing representations with which to compare ragas in this tradition. Finally the article by $\mathrm{P}$. Rao, J. Ch. Ross, K. K. Ganguli, V. Pandit, V. Ishwar, A. Bellur and H. Murthy is an initial attempt at analyzing melodic motives in Indian art music, proposing appropriate representations for them and identifying problems to be worked on.

The eight articles presented in this issue offer a representative view of what is being done in the field of music information research related to Turkish-makam and Indian art music traditions and at the same time they identify many relevant research problems that should help advance the field of music information research in the near future.

\author{
Xavier Serra \\ Music Technology Group \\ Universitat Pompeu Fabra, Barcelona
}

